\title{
OPEN Relationship between basal sodium intake and the effects of dapagliflozin in albuminuric diabetic kidney disease
}

\author{
Sho Kinguchi ${ }^{1}$, Hiromichi Wakui ${ }^{1}$, Yuzuru Ito $^{2}$, Yoshinobu Kondo ${ }^{2}$, Kengo Azushima ${ }^{1}$, \\ Uru Osada ${ }^{3}$, Tadashi Yamakawa ${ }^{4}$, Tamio Iwamoto ${ }^{5}$, Jun Yutoh ${ }^{6}$, Toshihiro Misumi ${ }^{7}$, \\ Gen Yasuda ${ }^{8}$, Taishi Yoshii ${ }^{9}$, Kotaro Haruhara ${ }^{1}$, Yusuke Kobayashii ${ }^{1,10}$, Takeharu Yamanaka ${ }^{7}$, \\ Yasuo Terauchi $^{\circledR \otimes}$ \& Kouichi Tamura ${ }^{1 \bowtie}$
}

We investigated the impact of basal dietary sodium intake on the dapagliflozin-induced changes in albuminuria and blood pressure (BP) measured at home in patients with diabetic kidney disease (DKD). This was a secondary analysis of the Y-AIDA Study, in which DKD patients with estimated glomerular filtration rate (eGFR) $\geq 45 \mathrm{ml} / \mathrm{min} / 1.73 \mathrm{~m}^{2}$ and urinary albumin-to-creatinine ratio (UACR) $\geq 30 \mathrm{mg} / \mathrm{g}$ creatinine were administered dapagliflozin for 24 weeks, and dapagliflozin significantly improved albuminuria levels and home BP profiles. The effects on UACR, home-measured BP, and eGFR were compared between high- and low-sodium intake groups (HS and LS groups), which were created using baseline urinary sodium-to-creatinine ratio of 84 participants with available basal sodium-tocreatinine ratios. At baseline, clinic-/home-measured BPs, UACR, and eGFR, were comparable in the two groups. After 24 weeks, the reductions from baseline in In-UACR were comparable in the two groups. In contrast, the reductions in evening home systolic BP and eGFR from baseline were larger in HS than in LS (BP: $-13 \pm 2.08$ vs. $-6 \pm 1.88, P=0.020$; eGFR: $-3.33 \pm 1.32$ vs. $0.37 \pm 1.29$, $P=0.049$ ). The home BP-lowering effects of dapagliflozin are larger in HS than LS, concomitant with a larger reduction in eGFR, suggesting a dapagliflozin-induced improvement in glomerular relative hyperfiltration in HS.

Diabetic kidney disease (DKD) is a common comorbidity in patients with type 2 diabetes mellitus (T2DM), which is the leading cause of end-stage renal disease $(\mathrm{ESRD})^{1,2}$. DKD is conventionally thought to predominantly affect the glomerulus, through intraglomerular hypertension, which promotes glomerulosclerosis and inflammation especially in the context of systemic hypertension ${ }^{3,4}$. From a renal mechanistic perspective, sodium plays an important role in the pathogenesis of DKD. High dietary sodium intake and volume expansion are risk factors for hypertension, which promotes the progression of $\mathrm{DKD}^{3,5}$. Furthermore, aberrant proximal tubular handling of sodium plays an important role in the pathogenesis of glomerular hyperfiltration ${ }^{6}$, which contributes to the increase in urinary albumin excretion and the progressive decline in renal function ${ }^{7}$.

\footnotetext{
${ }^{1}$ Department of Medical Science and Cardiorenal Medicine, Yokohama City University Graduate School of Medicine, 3-9 Fukuura, Kanazawa-ku, Yokohama 236-0004, Japan. 2Department of Endocrinology and Metabolism, Yokohama City University Graduate School of Medicine, 3-9 Fukuura, Kanazawa-ku, Yokohama 236-0004, Japan. ${ }^{3}$ Department of Diabetes and Endocrinology, Saiseikai Yokohama South Hospital, Yokohama, Japan. 'Department of Endocrinology and Diabetes, Yokohama City University Center Hospital, Yokohama, Japan. ${ }^{5}$ Department of Nephrology and Hypertension, Saiseikai Yokohama South Hospital, Yokohama, Japan. ${ }^{6}$ Department of Nephrology and Hypertension, Yokohama Minami Kyousai Hospital, Yokohama, Japan. ${ }^{7}$ Department of Biostatistics and Epidemiology, Yokohama City University Graduate School of Medicine, Yokohama, Japan. ${ }^{8}$ Department of Nephrology and Hypertension, Yokohama City University Center Hospital, Yokohama, Japan. ${ }^{9}$ Department of Endocrinology and Metabolism, Yokohama Minami Kyousai Hospital, Yokohama, Japan. ${ }^{10}$ Center for Novel and Exploratory Clinical Trials (Y-NEXT), Yokohama City University, Yokohama, Japan.『email: hiro1234@yokohama-cu.ac.jp; terauchi@yokohama-cu.ac.jp; tamukou@ med.yokohama-cu.ac.jp
} 
High sodium intake is associated with high blood pressure (BP), a risk factor for cardiovascular and renal diseases ${ }^{8}$, and the restriction of sodium intake lowers $\mathrm{BP}^{9-11}$. High sodium intake also causes resistant hypertension ${ }^{12}$. Some previous studies have also demonstrated that many Japanese patients with DKD have salt-sensitive hypertension ${ }^{13}$. Furthermore, high dietary sodium intake is associated with a high incidence of cardiovascular disease in T2DM patients ${ }^{14}$. The renal and cardiovascular protective effects of conventional therapy, which includes an angiotensin receptor blocker (ARB), were superior to those of non-renin-angiotensin system (RAS) inhibitor-based therapy in T2DM patients with high sodium intake ${ }^{15}$.

Dapagliflozin is a sodium-glucose cotransporter-2 (SGLT2) inhibitor that increases urinary glucose excretion and reduces hemoglobin $\mathrm{A} 1 \mathrm{c}(\mathrm{HbA1c})^{16}$. SGLT2 inhibitors are also reported to have pleiotropic effects, including reductions in body mass, $\mathrm{BP}$, and urinary albumin excretion, which have cardio-renal protective effects ${ }^{16-20}$. The inhibition of SGLT2 also increases urinary sodium excretion, and this natriuretic effect of SGLT2 inhibitors may be clinically important ${ }^{3,21,22}$. Recently, we reported that dapagliflozin improves albuminuria and the BP profile assessed at home, and the latter is associated with the amelioration of albuminuria in Japanese DKD patients ${ }^{23}$. Therefore, we hypothesized that the improvements in albuminuria and home BP profile that are associated with dapagliflozin may be more pronounced in Japanese DKD patients with high sodium intake.

The purpose of the present study was to investigate the impacts of basal dietary sodium intake, estimated using urinary sodium excretion, on the improvements in urinary albumin-to-creatinine ratio (UACR) and home BP associated with the use of dapagliflozin in Japanese DKD patients. To this end, data from the Yokohama Add-on Inhibitory efficacy of Dapagliflozin on Albuminuria in Japanese patients with type 2 diabetes study (Y-AIDA study) ${ }^{23}$ were analyzed.

\section{Methods}

Study design. We conducted a secondary analysis of data from the Y-AIDA study ${ }^{23}$, which was a prospective, multi-center, single-arm study that was performed as previously described ${ }^{23}$. Briefly, 86 T2DM patients with HbA1c 7.0-10.0\%, estimated glomerular filtration rate (eGFR) $\geq 45 \mathrm{ml} / \mathrm{min} / 1.73 \mathrm{~m}^{2}$, and urine albumin-to-creatinine ratio (UACR) $\geq 30 \mathrm{mg} / \mathrm{g}$ creatinine $(\mathrm{gCr})$ were enrolled, and 85 of these patients were administered addon dapagliflozin for 24 weeks ${ }^{23}$. This secondary analysis included 84 participants whose basal urinary sodiumto-creatinine ratios were available. The study complied with the ethical principles of the Declaration of Helsinki. Ethical approval was obtained from the ethical review boards of Yokohama City University (B150305009), Yokohama City University Center Hospital, Saiseikai Yokohama South Hospital and Yokohama Minami Kyousai Hospital. The study is registered in the UMIN Clinical Trials Registry (UMIN000018930; http://www.umin.ac.jp/ctr/ index-j.htm). All the participants provided written informed consent prior to their participation.

Physical findings and laboratory measurements. Body mass, body mass index (BMI), and BP were measured, and blood and urine sampling was performed in the clinic in the fasted state, at baseline (0 weeks) and after 8 weeks, 16 weeks, and 24 weeks of treatment, as described previously ${ }^{23}$. Urinalysis was performed in spot urine samples at the applicable visits. At baseline, urine sodium $(\mathrm{mEq} / \mathrm{L})$ was measured using the electrode method, and urine creatinine $(\mathrm{mg} / \mathrm{dL})$ was measured using an enzymatic method, by SRL Inc. (Tokyo, Japan).

Home BP and nocturnal home BP measurements. Home BP and nocturnal home BP were measured using a validated and internet-interfaced BP monitor (HEM-7252G-HP, Omron Corporation, Kyoto), as previously described $^{23}$. The home BP monitoring device enabled automatic transmission of all home BP readings to a server after each measurement, using a mobile telecommunication system (MedicalLINK), to allow accurate analysis of the home BP profile ${ }^{24,25}$. Three home BP readings were taken at 1 -min intervals in a sitting position in the morning and evening during the 7 -day study periods before the 0 -week and 24 -week visits. Morning BP was measured before breakfast, within $1 \mathrm{~h}$ of waking, and before taking anti-hypertensive medication. Evening BP was measured before going to bed. The morning and evening home BPs were defined as the mean BP value during the mornings and evenings of the 7-day study periods, which were the means of the three readings obtained in the morning and evening of each day. The HEM-7252G-HP monitor can also record BP readings at fixed times, and was pre-set to record nocturnal home BP measurements at 02:00, 03:00, 04:00, and 05:00 h. Nocturnal home BPs were also measured during the 7-day study period before the 0 - and 24 -week visits. Nocturnal home $\mathrm{BP}$ was defined as the mean nighttime BP during the 7-day study periods, which represented the mean of four readings per night.

Statistical analysis. To assess the effects of baseline dietary sodium intake on clinical variables during add-on dapagliflozin administration, participants were allocated to two groups according to their median urinary sodium-to-creatinine ratio at baseline. The median urinary sodium-to-creatinine ratio at baseline was 1.14 $\left(\times 10^{2} \mathrm{mEq} / \mathrm{g} \mathrm{Cr}\right.$ ) (interquartile range (IQR): $\left.0.815,1.595\right)$. Therefore, we allocated participants with urinary sodium-to-creatinine ratios $>1.14$ to the high-sodium intake group (HS group) and the other participants, who had urinary sodium-to-creatinine ratios $<1.14$, to the low-sodium intake group (LS group). Their estimated daily salt intakes (g/day) were also calculated using the Tanaka formula ${ }^{26}$. The Tanaka formula is as follows: 24 -h urinary sodium $(\mathrm{Na})$ excretion $(\mathrm{mEq} /$ day $)=21.98 \times\{[$ Spot urinary sodium concentration $(\mathrm{mEq} / \mathrm{L}) \div($ Spot urinary creatinine concentration $(\mathrm{mg} / \mathrm{dL}) \times 10)] \times$ predicted 24 -h urinary creatinine excretion $(\operatorname{PrUCr} 24 \mathrm{~h})(\mathrm{mg} /$ day) $\}^{0.392}$; PrUCr24h $(\mathrm{mg} /$ day $)=14.89 \times$ weight $(\mathrm{kg})+16.14 \times$ height $(\mathrm{cm})-2.04 \times$ age $($ years $)-2,244.45$. Estimated daily salt intake $(\mathrm{g} /$ day $)=24$-h urinary sodium excretion $(\mathrm{mEq} /$ day $) \div 17$.

Furthermore, participants were allocated to two groups according to median estimated daily salt intake (g/ day) calculated using the Tanaka formula at baseline. Median estimated daily salt intake at baseline was 9.213 (g/day) (IQR: 8.063, 10.446). Therefore, we allocated participants with estimated daily salt intake $>9.213$ to the 


\begin{tabular}{|c|c|c|c|}
\hline Variable & $\begin{array}{l}\text { HS group } \\
\mathrm{N}=42\end{array}$ & $\begin{array}{l}\text { LS group } \\
\mathrm{N}=42\end{array}$ & $P$ value \\
\hline Urinary $\mathrm{Na} / \mathrm{Cr}$ ratio $\left(\times 10^{2} \mathrm{mEq} / \mathrm{g} \mathrm{Cr}\right)$ & $1.85 \pm 0.11$ & $0.76 \pm 0.05$ & $<0.001$ \\
\hline Estimated daily salt intake (g/day) & $10.88 \pm 0.29$ & $7.80 \pm 0.27$ & $<0.001$ \\
\hline Age (years) & $65.6 \pm 1.5$ & $64.9 \pm 1.6$ & 0.772 \\
\hline Sex (male/female) & $28 / 14$ & $36 / 6$ & 0.071 \\
\hline Body mass index $\left(\mathrm{kg} / \mathrm{m}^{2}\right)$ & $27.5 \pm 0.8$ & $26.8 \pm 0.6$ & 0.512 \\
\hline Duration of diabetes (years) & $14.8 \pm 1.6$ & $10.3 \pm 1.2$ & 0.027 \\
\hline Hypertension n (\%) & $36(85.7)$ & $37(92.5)$ & 0.483 \\
\hline Dyslipidemia n (\%) & $36(85.7)$ & $35(87.5)$ & 1.000 \\
\hline Hyperuricemia n (\%) & $6(14.3)$ & $13(32.5)$ & 0.068 \\
\hline Angina pectoris $\mathrm{n}(\%)$ & $5(12.2)$ & $4(9.5)$ & 0.738 \\
\hline Myocardial infarction n (\%) & $2(4.9)$ & $0(0.0)$ & 0.241 \\
\hline Stroke n (\%) & $4(9.8)$ & $7(16.7)$ & 0.520 \\
\hline \multicolumn{4}{|l|}{ Clinic blood pressure } \\
\hline $\mathrm{SBP}(\mathrm{mmHg})$ & $144 \pm 3.3$ & $140 \pm 2.9$ & 0.404 \\
\hline $\mathrm{DBP}(\mathrm{mmHg})$ & $79 \pm 2.0$ & $78 \pm 1.9$ & 0.845 \\
\hline \multicolumn{4}{|l|}{ Glucose metabolism } \\
\hline Fasting blood glucose (mg/dl) & $156.7 \pm 6.1$ & $158.9 \pm 5.6$ & 0.792 \\
\hline HbAlc (\%) & $8.0 \pm 0.1$ & $7.7 \pm 0.1$ & 0.029 \\
\hline \multicolumn{4}{|l|}{ Renal function } \\
\hline eGFR $\left(\mathrm{ml} / \mathrm{min} / 1.73 \mathrm{~m}^{2}\right)$ & $69.8 \pm 2.7$ & $65.0 \pm 2.7$ & 0.219 \\
\hline Median UACR (mg/g Cr) (interquartile range) & $\begin{array}{l}199.5 \\
(61.4,629.0)\end{array}$ & $\begin{array}{l}102.5 \\
(40.1,666.0)\end{array}$ & 0.310 \\
\hline
\end{tabular}

Table 1. Baseline characteristics of the participants. Values are mean \pm standard error (SE). UACR is expressed as median (interquartile range). Analyses were performed using unpaired $t$-tests for continuous data and Fisher's exact tests for categorical data. Mann-Whitney $U$ test was used for analysis of UACR. HS group high sodium intake group, $L S$ group low sodium intake group, $\mathrm{Na}$ sodium, $\mathrm{Cr}$ creatinine, $S B P$ systolic blood pressure, $D B P$ diastolic blood pressure, $H b A 1 c$ glycated hemoglobin, $e G F R$ estimated glomerular filtration rate, $U A C R$ urine albumin-to-creatinine ratio.

high salt intake group, and those with estimated daily salt intake $<9.213$ to the low salt intake group. Changes in natural logarithm of UACR (ln-UACR), eGFR, and home BP from baseline were compared between the high salt intake and low salt intake groups.

The data in the text, figures, and tables are presented as mean \pm standard error (SE), percentage, or median (IQR). For statistical analyses of the differences between the high-sodium intake and low-sodium intake groups, unpaired $t$-tests was performed for continuous variables and Fisher's exact test was performed for categorical variables, at baseline and at each subsequent visit. Differences in UACR at baseline were analyzed using the Mann-Whitney $U$ test. Statistical analysis was performed using SAS version 9.4 (SAS Institute Japan) at the Department of Biostatistics and Epidemiology in Yokohama City University Graduate School of Medicine, and a value of $P<0.05$ was considered to represent statistical significance.

\section{Results}

Baseline patient characteristics of the HS and LS groups. Eighty-five participants were administered add-on dapagliflozin for 24 weeks in the Y-AIDA study, as described previously ${ }^{23}$. The participants were divided into two groups (the HS and LS groups) according to their median basal urinary sodium-to-creatinine ratio (Table 1,2$)$. The duration of diabetes was longer $(14.8 \pm 1.6$ vs. $10.3 \pm 1.2$ years, $P=0.027)$ and HbAlc was higher $(8.0 \pm 0.1$ vs. $7.7 \pm 0.1, P=0.029)$ in the HS group than in the LS group. The other parameters (body mass, BMI, UACR, and eGFR) were comparable in the two groups (Table 1). Median UACR was $199.5 \mathrm{mg} / \mathrm{g} \mathrm{Cr}$ (IQR: $61.4,629.0)$ in the HS group, and $102.5 \mathrm{mg} / \mathrm{g} \mathrm{Cr}$ (IQR: $40.1,666.0)$ in the LS group $(P=0.310)$. There were no significant differences in the concomitant use of antidiabetic agents or antihypertensive agents, including RAS inhibitors and diuretics, between the two groups (Table 2).

Effects of add-on dapagliflozin therapy on renal endpoints in the HS and LS groups. The natural logarithm of UACR (ln-UACR) and eGFR were similar at each visit in the two groups (Fig. 1a,b). However, regarding the changes from baseline, the reduction in ln-UACR from baseline tended to be larger at week 8 $(-0.36 \pm 0.11$ vs. $-0.09 \pm 0.11, P=0.092)$, and was significantly larger at week $16(-0.54 \pm 0.10$ vs. $-0.10 \pm 0.13$, $P=0.010)$ in the HS group. However, after 24 weeks, the reductions from baseline in ln-UACR were comparable in the two groups $(-0.46 \pm 0.11$ vs. $-0.27 \pm 0.12, P=0.237$; Fig. 1c). The reduction in eGFR from baseline was similar at week $8(-2.36 \pm 1.18$ vs. $-2.12 \pm 1.29, P=0.890)$, whereas the reductions in eGFR at week 16 and week 24 from baseline were larger in the HS group (week 16: $-3.66 \pm 1.17$ vs. $0.14 \pm 1.29, P=0.033$; week $24:-3.33 \pm 1.32$ vs. $0.37 \pm 1.29, P=0.049$; Fig. $1 \mathrm{~d})$. 


\begin{tabular}{|c|c|c|c|}
\hline Variable & $\begin{array}{l}\text { HS group } \\
\mathrm{N}=42\end{array}$ & $\begin{array}{l}\text { LS group } \\
\mathrm{N}=42\end{array}$ & $P$ value \\
\hline \multicolumn{4}{|l|}{ Antidiabetic agents } \\
\hline Insulin n (\%) & $14(36.8)$ & $13(37.1)$ & 1.000 \\
\hline Biguanides n (\%) & $28(73.7)$ & $20(57.1)$ & 0.149 \\
\hline DPP-4 inhibitors n (\%) & $22(57.9)$ & $24(68.6)$ & 0.467 \\
\hline Sulfonylureas n (\%) & $15(39.5)$ & $8(22.9)$ & 0.141 \\
\hline a-glucosidase inhibitors n (\%) & $6(15.8)$ & $11(31.4)$ & 0.166 \\
\hline Thiazolidinediones n (\%) & $4(10.5)$ & $8(22.9)$ & 0.211 \\
\hline Glinides n (\%) & $3(7.9)$ & $4(11.4)$ & 0.704 \\
\hline GLP1 agonists n (\%) & $3(7.9)$ & $5(14.3)$ & 0.468 \\
\hline \multicolumn{4}{|l|}{ Antihypertensive agents } \\
\hline \multicolumn{4}{|l|}{ RAS inhibitors } \\
\hline Angiotensin II receptor blockers n (\%) & $28(66.7)$ & $29(69.0)$ & 1.000 \\
\hline Angiotensin-converting enzyme inhibitors n (\%) & $3(7.1)$ & $1(2.4)$ & 0.616 \\
\hline Calcium-channel blockers n (\%) & $27(64.3)$ & $25(59.5)$ & 0.823 \\
\hline Diuretics n (\%) & $6(14.3)$ & $4(9.5)$ & 0.738 \\
\hline al-blockers n (\%) & $1(2.4)$ & $1(2.4)$ & 1.000 \\
\hline$\beta$-blockers n (\%) & $4(9.5)$ & $3(7.1)$ & 1.000 \\
\hline Aldosterone antagonist n (\%) & $1(2.4)$ & $2(4.8)$ & 1.000 \\
\hline a2-agonists n (\%) & $0(0.0)$ & $2(4.8)$ & 0.494 \\
\hline
\end{tabular}

Table 2. Baseline medications of the participants. Analyses were performed using unpaired $t$-tests for continuous data and Fisher's exact tests for categorical data. HS group high sodium intake group, LS group low sodium intake group, DPP-4 dipeptidyl peptidase 4, GLP1 glucagon-like peptide 1, RAS renin-angiotensin system.

Effects of add-on dapagliflozin therapy on the glucose metabolism, clinic-measured blood pressure, body mass, and body mass index of the HS and LS groups. Although HbAlc was significantly higher at baseline in the HS group than in the LS group (7.99 \pm 0.12 vs. $7.66 \pm 0.09, P=0.029$; Fig. 2a), there were no significant differences in HbAlc at weeks 8, 16, and 24 between the two groups. In addition, the reduction in HbAlc from baseline was significantly larger at week 8 in the HS group $(-0.58 \pm 0.08$ vs. $-0.30 \pm 0.09, P=0.028$; Fig. $2 \mathrm{~b}$ ). At weeks 16 and 24 , the reductions in $\mathrm{HbA1c}$ from baseline were similar in the two groups (week $16:-0.57 \pm 0.09$ vs. $-0.32 \pm 0.09, P=0.057$; week $24:-0.59 \pm 0.10$ vs. $-0.40 \pm 0.08, P=0.148$; Fig. 2b). However, there were no significant differences in fasting blood glucose (FBG), clinic systolic BP, clinic diastolic BP, body mass, and BMI between the two groups at each visit (Fig. 2c,e). Similarly, the reductions in these parameters from baseline were comparable at each visit in the two groups (Fig. 2d,f).

Effects of add-on dapagliflozin therapy on home-measured blood pressure in the HS and LS groups. At baseline, the morning, evening, and nocturnal home systolic/diastolic BPs were similar in the two groups (morning systolic BP: $138 \pm 1.94$ vs. $136 \pm 2.60 \mathrm{mmHg}, P=0.518$; morning diastolic BP: $82 \pm 1.61$ vs. $83 \pm 1.65 \mathrm{mmHg}, P=0.635$; evening systolic $B P: 137 \pm 2.86$ vs. $134 \pm 2.78 \mathrm{mmHg}, P=0.448$; evening diastolic BP: $79 \pm 1.77$ vs. $79 \pm 1.50 \mathrm{mmHg}, P=0.924$; nocturnal systolic $B P$ : $124 \pm 2.43$ vs. $125 \pm 2.35 \mathrm{mmHg}, P=0.727$; nocturnal diastolic BP: $72 \pm 1.73$ vs. $75 \pm 1.30 \mathrm{mmHg}, P=0.268$; Fig. 3a,c,e,g,i,k). After 24 weeks of dapagliflozin treatment, compared with the LS group, the reduction in evening systolic BP from baseline was significantly larger $(-13 \pm 2.08$ vs. $-6 \pm 1.88, P=0.020$; Fig. $3 \mathrm{f})$, and the reduction in evening diastolic $\mathrm{BP}$ tended to be larger $(-5 \pm 1.17$ vs. $-3 \pm 0.99, P=0.078$; Fig. $3 \mathrm{~h}$ ), in the HS group. In contrast, the changes in morning and nocturnal BP from baseline were comparable at week 24 in the two groups (Fig. 3b,d,j,l).

Effects of add-on dapagliflozin therapy on renal endpoints and home-measured blood pressure in the high salt intake and low salt intake groups based on the Tanaka formula. Estimation of salt intake by spot urine was calculated using the Tanaka formula ${ }^{26}$. This formula can also be used to estimate salt intake in patients with $\mathrm{CKD}^{27}$. To assess the effects of baseline dietary salt intake on clinical variables during administration of add-on dapagliflozin, participants were divided into two groups (high and low salt intake) according to median estimated daily salt intake calculated using the Tanaka formula at baseline. Median estimated daily salt intake was 10.45 (IQR: $9.65,12.15$ ) and 8.06 (IQR: 7.16, 8.79) in the high and low salt intake groups, respectively $(P<0.001)$. Reductions in ln-UACR from baseline were consistent with the above results of the comparison of reductions in $\ln$-UACR between the HS and LS groups based on urinary sodiumto-creatinine ratio at baseline (Fig. 4a). Reductions in eGFR from baseline were similar at week 8 in both groups $(-3.43 \pm 1.11$ vs. $-1.05 \pm 1.32, P=0.173)$, whereas reductions in eGFR at week 16 and week 24 from baseline were larger in the high salt intake group (week $16:-3.70 \pm 1.12$ vs. $0.18 \pm 1.33, P=0.029$; week $24:-3.85 \pm 1.18$ vs. $0.89 \pm 1.38, P=0.011$; Fig. 4b). 
a

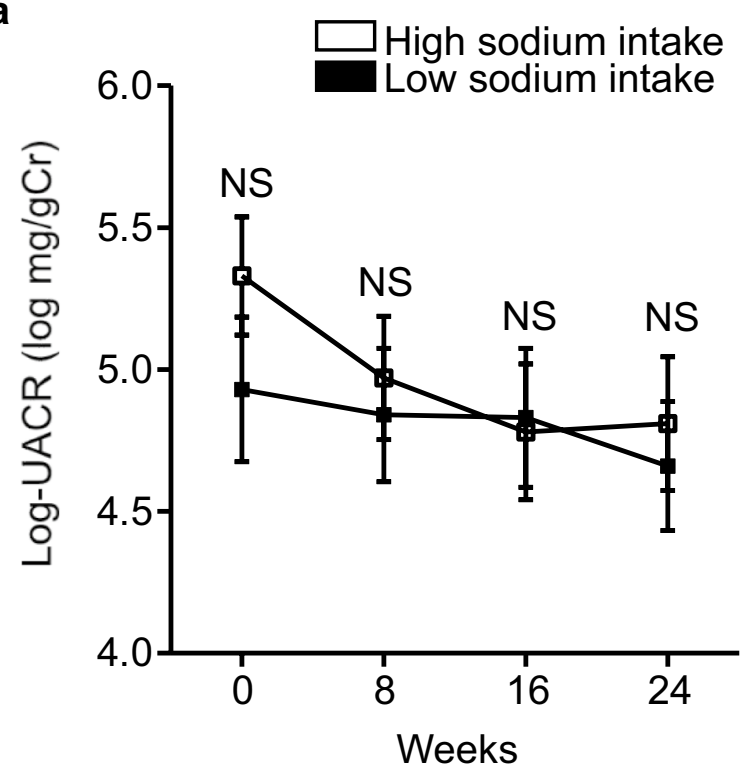

b

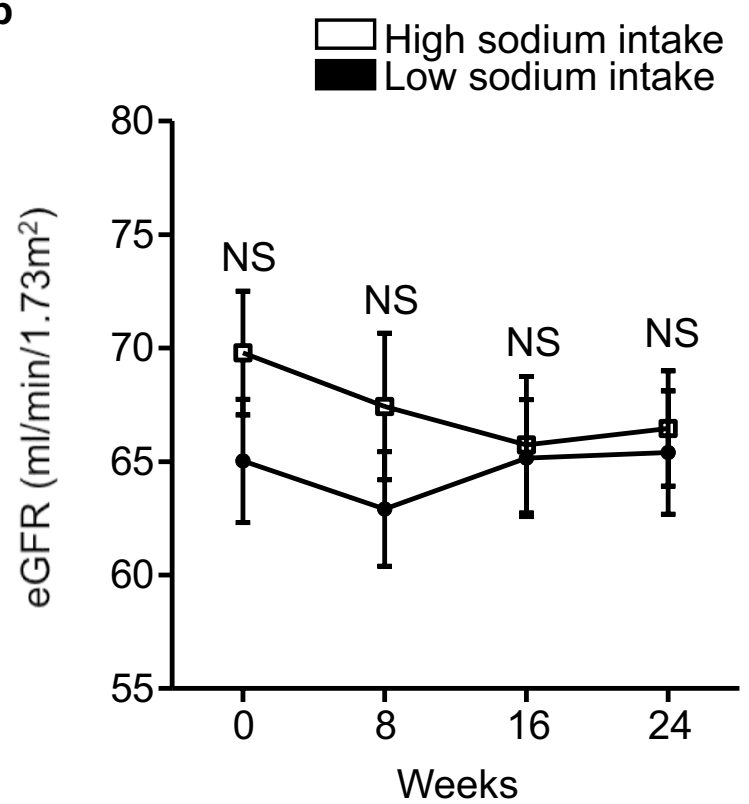

C

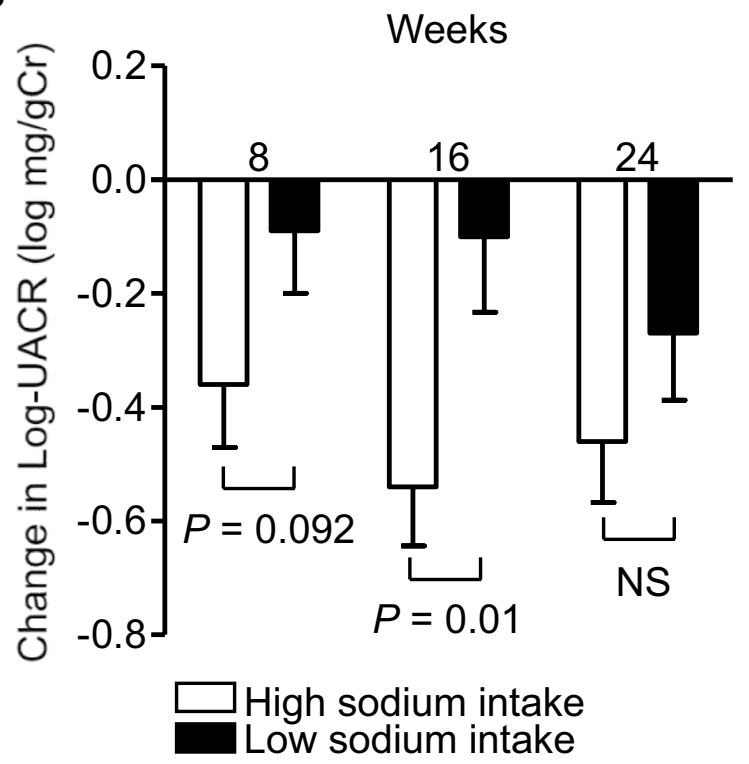

d

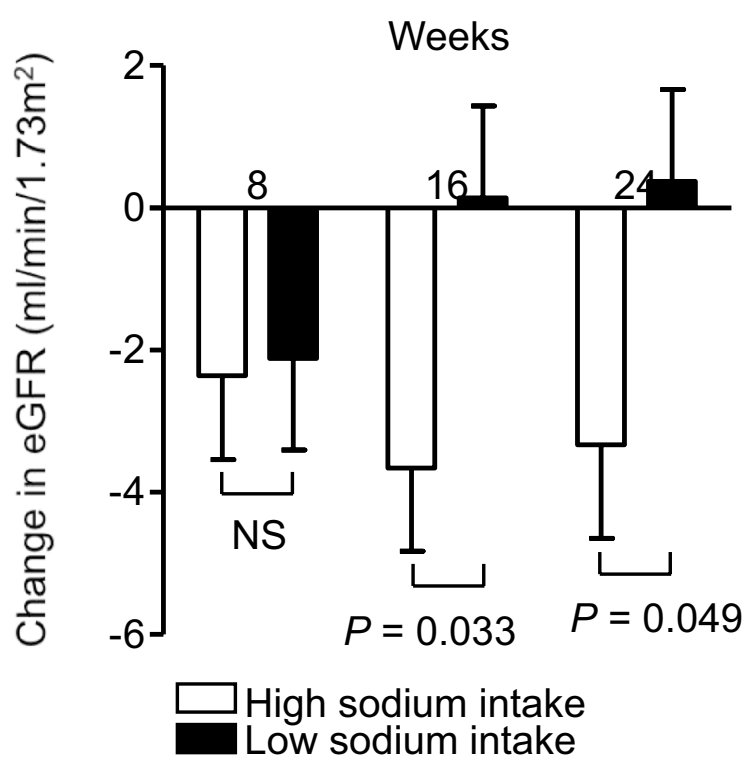

Figure 1. Effects of add-on dapagliflozin therapy on renal endpoints in the HS and LS groups. (a) Natural logarithm of urine albumin-to-creatinine ratio (ln-UACR), and (b) estimated glomerular filtration (eGFR) in dapagliflozin-treated patients during the 24 -week study period. Change in (c) ln-UACR, and (d) eGFR during the 24-week study period. Eighty-four DKD patients were allocated to a high sodium intake (HS) group (open square) or a low sodium intake (LS) group (filled square), according to their median urinary sodiumto-creatinine ratio at baseline. Values are mean \pm SE. The differences in the effects of dapagliflozin treatment between the two groups were analyzed using unpaired $t$-tests at each visit. NS not significant.

After 24 weeks of dapagliflozin treatment, compared with the low salt intake group, reduction in evening systolic BP from baseline was significantly larger $(-12 \pm 2.10$ vs. $-6 \pm 1.89, P=0.037$; Fig. $4 \mathrm{~d})$ in the high salt intake group. In contrast, changes in morning and nocturnal systolic BP from baseline were comparable at week 24 in both groups (Fig. 4c,e). 
a

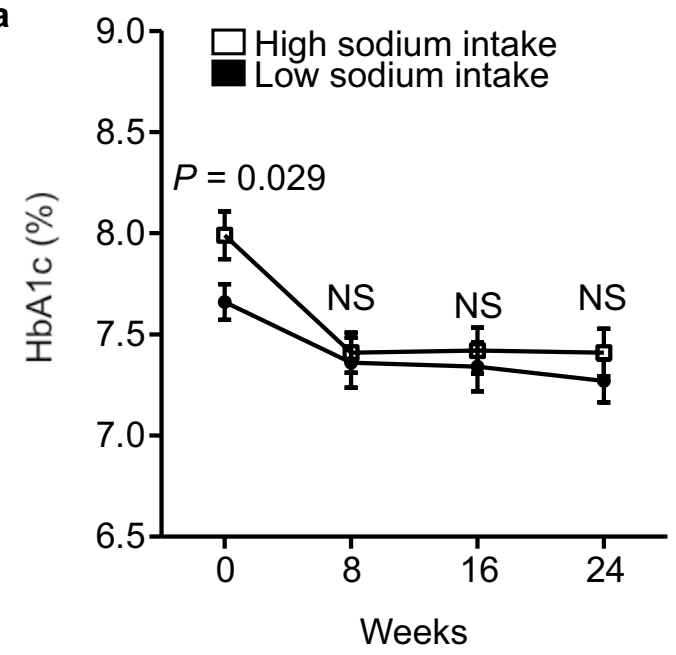

C

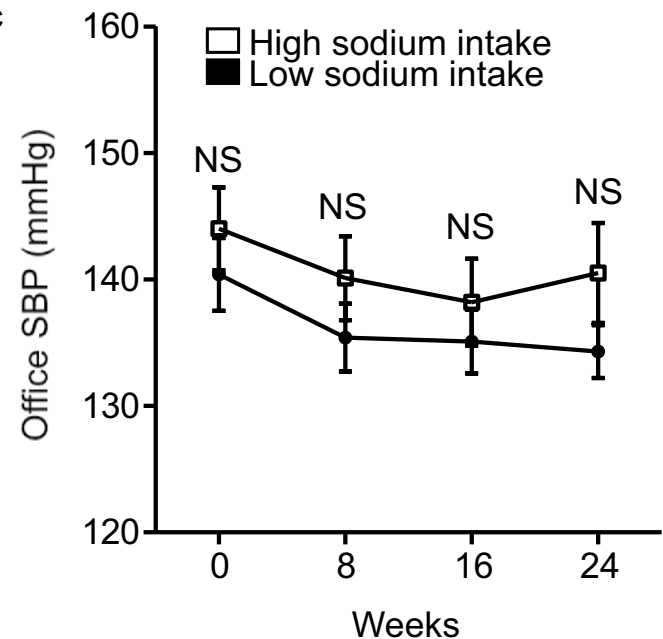

e

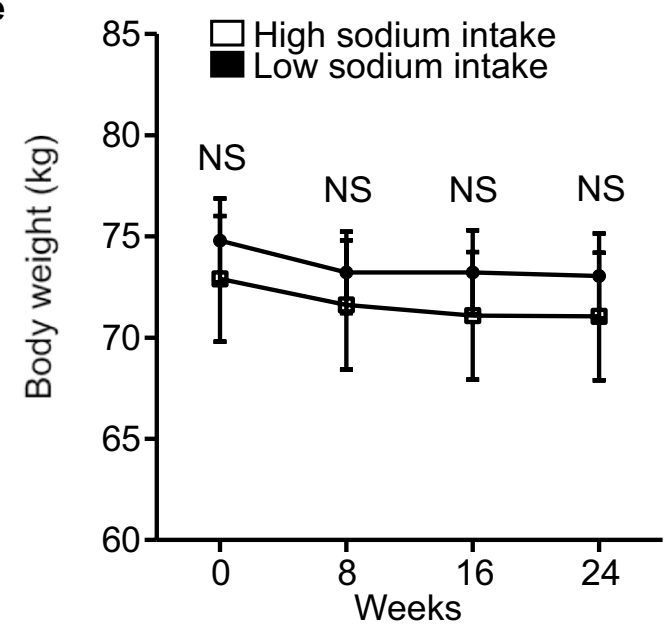

b

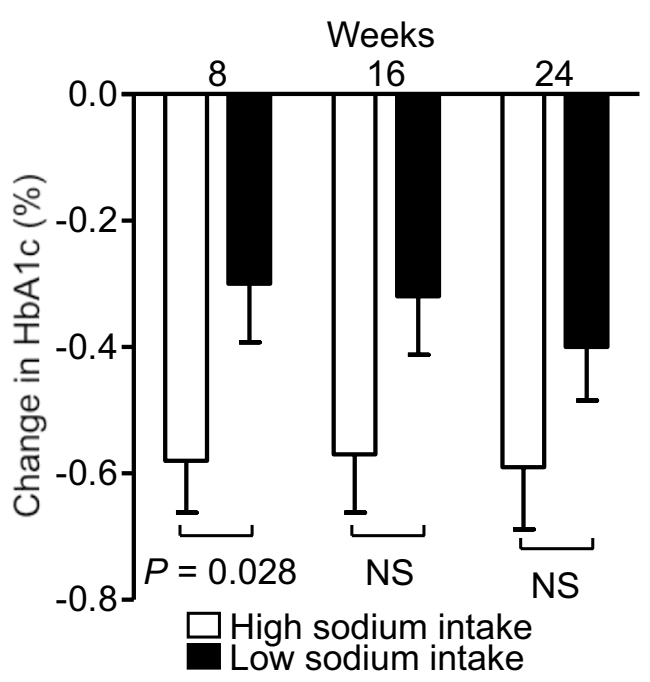

d

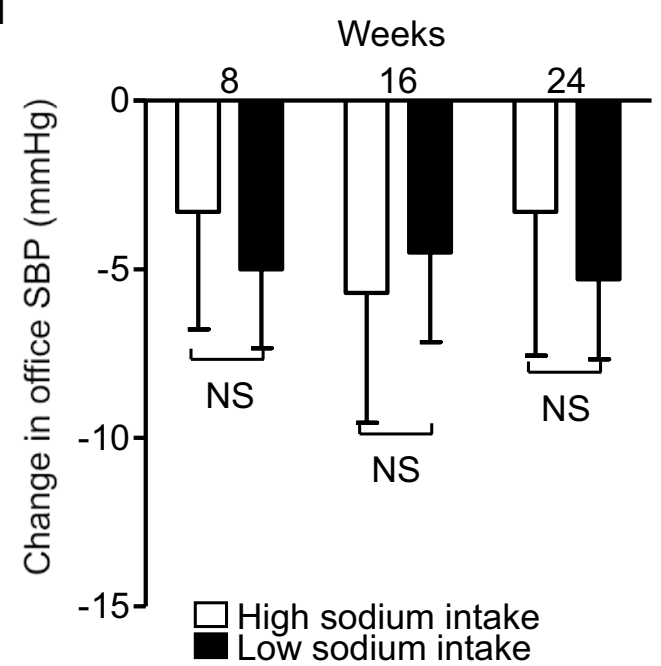

$\mathbf{f}$

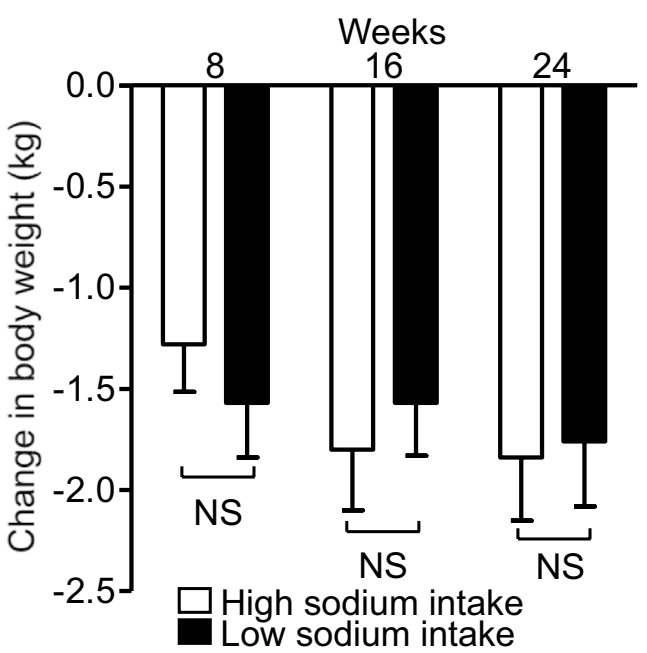

Figure 2. Effects of add-on dapagliflozin therapy on the glucose metabolism, clinic-measured blood pressure, body mass, and body mass index of the HS and LS groups. (a) HbAlc, (c) fasting blood glucose (FBG), (e) clinic systolic blood pressure (SBP), (g) clinic diastolic blood pressure (DBP), (i) body mass, and (k) body mass index (BMI) in dapagliflozin-treated patients during the 24-week study period. Change in (b) HbAlc, (d) FBG, (f) clinic SBP, (h) clinic DBP, (j) body mass, and (l) BMI during the 24-week study period. Eighty-four DKD patients were allocated to a high sodium intake (HS) group (open square) or a low sodium intake (LS) group (filled square), according to their median urinary sodium-to-creatinine ratio at baseline. Values are mean \pm SE. The differences in the effects of dapagliflozin treatment between the two groups were analyzed using unpaired $t$-tests at each visit. NS, not significant. 
a

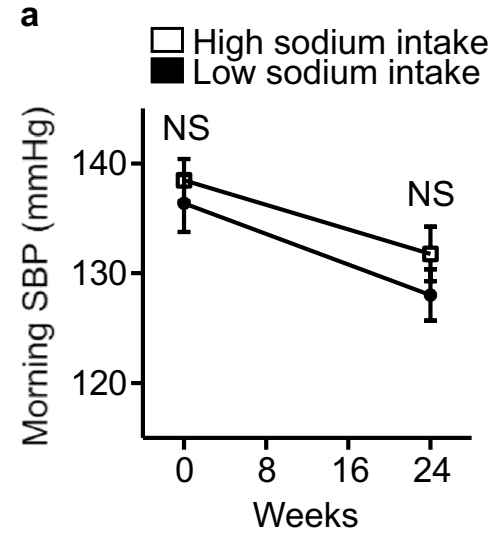

b

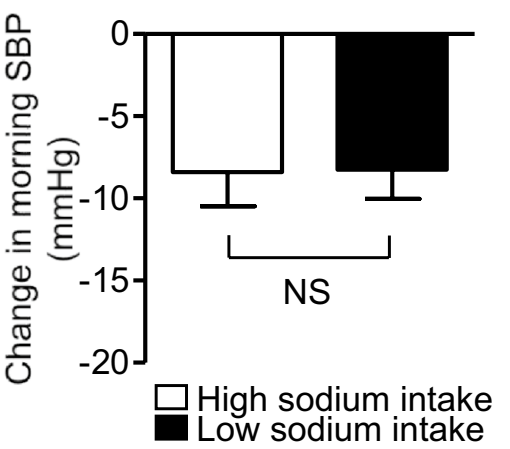

C

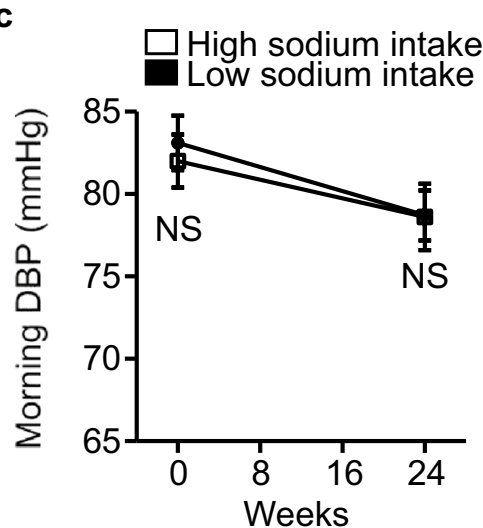

d

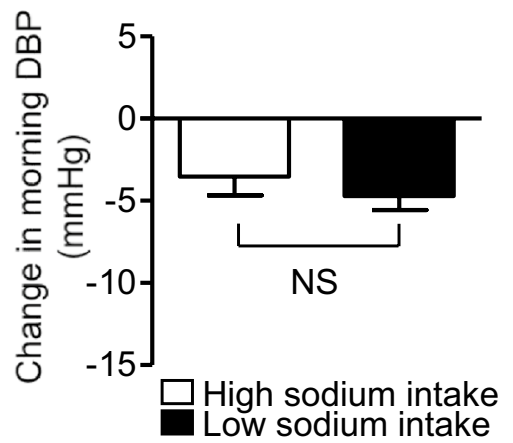

e

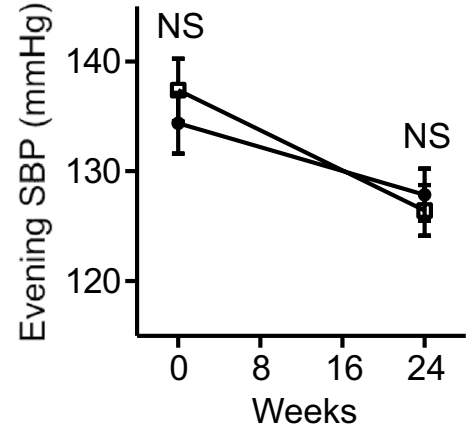

f

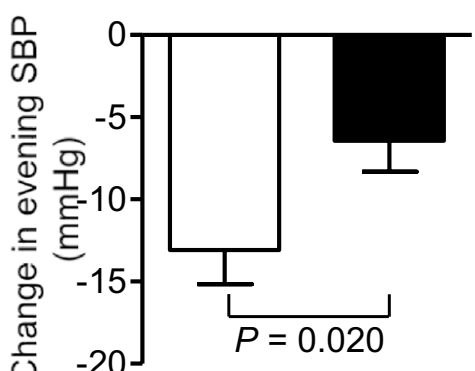

High sodium intake Low sodium intake

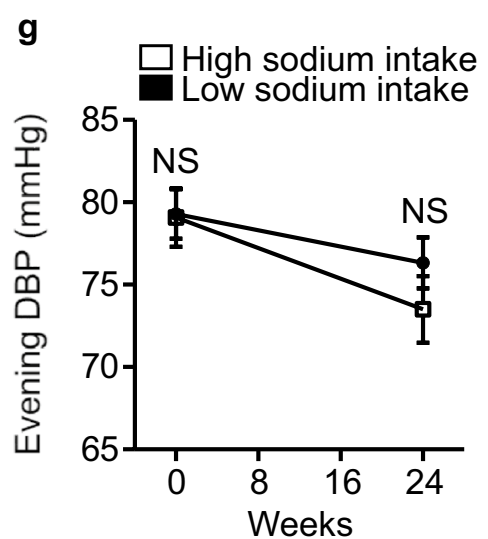

h

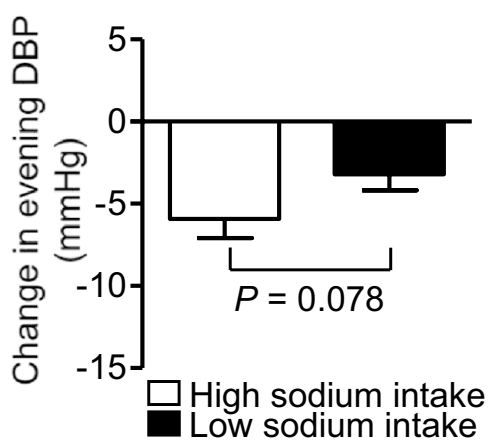

i

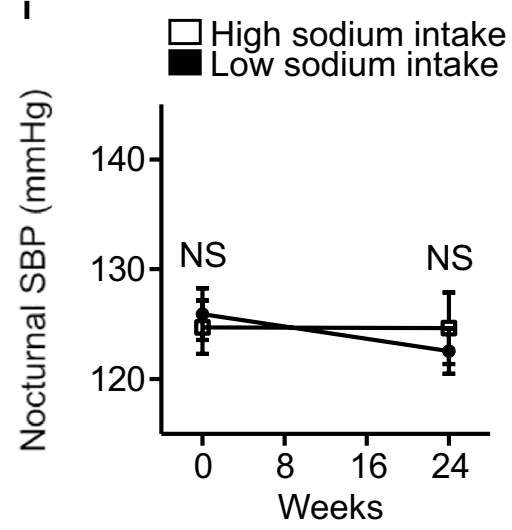

j

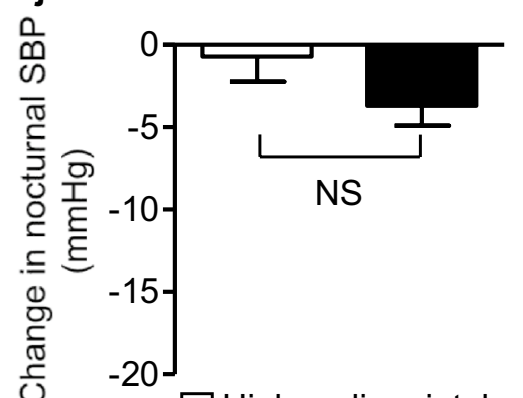

High sodium intake Low sodium intake

$\mathbf{k}$

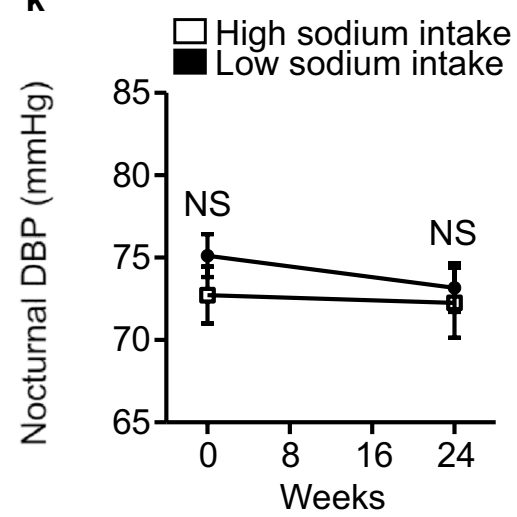

I

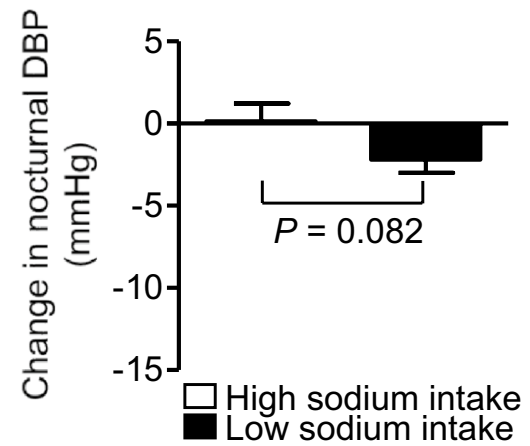

Figure 3. Effects of add-on dapagliflozin therapy on home-measured blood pressure in the HS and LS groups. Home blood pressure (BP) ((a) morning systolic BP (SBP), (c) morning diastolic BP (DBP), (e) evening SBP, (g) evening DBP, (i) nocturnal SBP, and (k) nocturnal DBP) in dapagliflozin-treated patients during the 24-week study period. Change in home BP ((b) morning SBP, (d) morning DBP, (f) evening SBP, (h) evening DBP, (j) nocturnal SBP, and (l) nocturnal DBP) during the 24-week study period. Eighty-four DKD patients were allocated to a high sodium intake (HS) group (open square) or a low sodium intake (LS) group (filled square), according to their median urinary sodium-to-creatinine ratio at baseline. Values are mean $\pm \mathrm{SE}$. The differences in the effects of dapagliflozin treatment between the two groups were analyzed using unpaired $t$-tests. NS, not significant. 
a

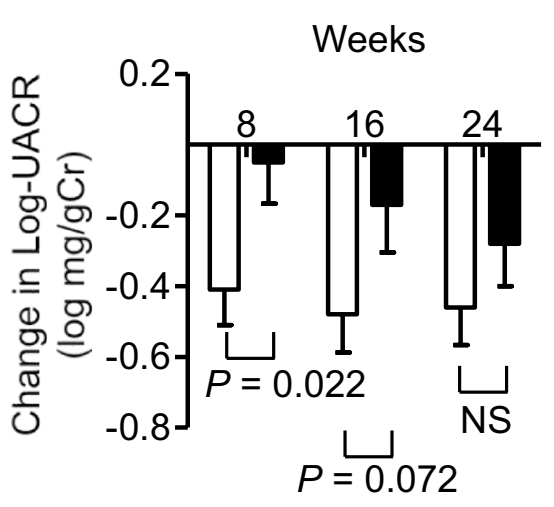

C

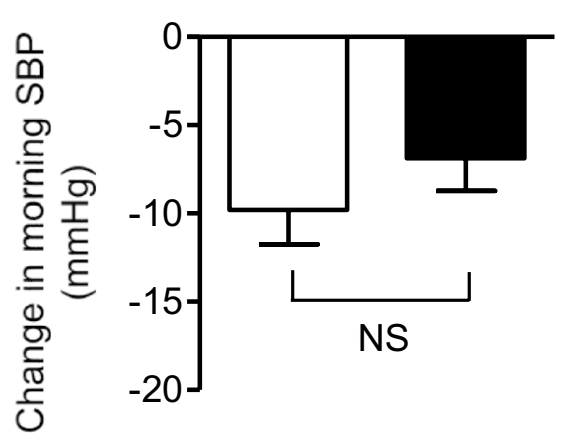

High salt intake Low salt intake b

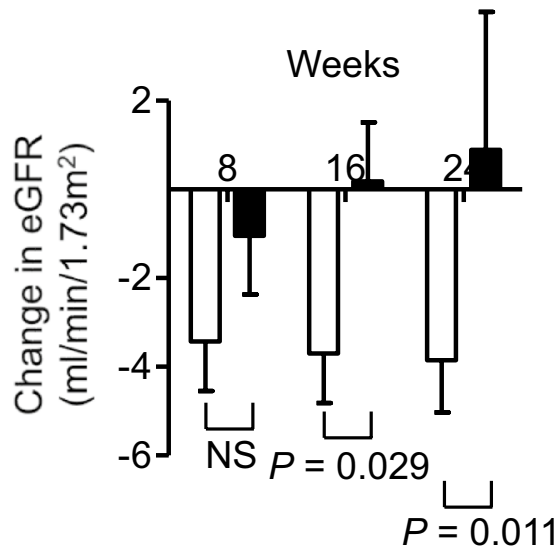

High salt intake

Low salt intake d

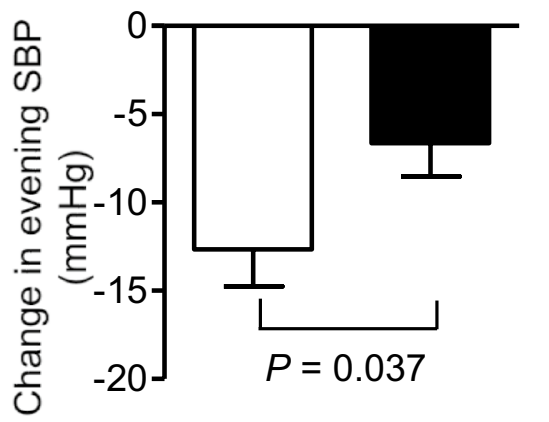

$\square$ High salt intake e

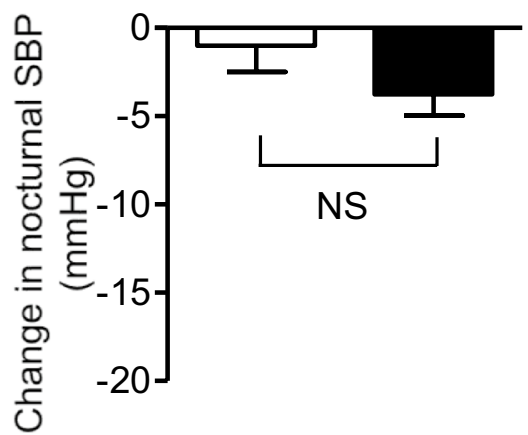

$\square$ High salt intake

Figure 4. Effects of add-on dapagliflozin therapy on renal endpoints and home-measured blood pressure in the high salt intake and low salt intake groups based on the Tanaka formula. Change in (a) natural logarithm of urine albumin-to-creatinine ratio (ln-UACR), and (b) estimated glomerular filtration (eGFR) during the 24-week study period. Change in home blood pressure (BP) ((c) morning systolic BP (SBP), (d) evening SBP, and (e) nocturnal SBP) during the 24-week study period. Eighty-four DKD patients were allocated to the high salt intake group (open square) or low salt intake group (filled square), according to median estimated daily salt intake calculated using the Tanaka formula at baseline. Values are presented as mean \pm SE. Differences in the effects of dapagliflozin treatment between the two groups were analyzed using unpaired $t$-tests at each visit. NS not significant.

\section{Discussion}

The present study is the first to demonstrate an impact of basal sodium intake on the non-clinic measured BPlowering effect of an SGLT2 inhibitor in albuminuric DKD patients. Four new findings were made in the present study. (i) Dapagliflozin was associated with reduction of albuminuria, regardless of basal sodium intake. (ii) The reduction in evening home systolic BP associated with dapagliflozin use was larger in the HS group than in the LS group. (iii) Dapagliflozin reduced clinic BP, and morning and nocturnal home BP, regardless of basal sodium intake. (iv) The reduction in eGFR from baseline associated with dapagliflozin use was larger in the HS group than in the LS group.

Many previous studies have shown that high sodium intake increases BP, which is a risk factor for cardiovascular and renal diseases ${ }^{8}$, and the restriction of sodium intake lowers $\mathrm{BP}^{9-11}$. High sodium intake also causes resistant hypertension ${ }^{12}$. Asian patients, including Japanese patients, have genetically high salt sensitivity and high dietary salt intake ${ }^{28,29}$. Several studies have also demonstrated that BP is salt sensitive in Japanese patients with type 2 diabetic nephropathy ${ }^{13}$. Furthermore, the BP-lowering effect of sodium restriction is larger in patients with diabetes mellitus $(\mathrm{DM})^{30}$. Published guidelines also recommend sodium intake to be limited to 2.0 g per 
day (equivalent to $5.0 \mathrm{~g}$ salt per day) for the management of hypertension ${ }^{12}$. However, the mean sodium intake is higher than that recommended for sodium restriction in the general Japanese population ${ }^{31}$.

The use of diuretics for the treatment of hypertension is effective in patients with DM, insulin resistance, or chronic kidney disease (CKD) who have high salt sensitivity, and recommended in patients who cannot restrict their sodium intake, have excess fluid volume indicated by findings such as edema, or have resistant hypertension $^{32,33}$. SGLT2 inhibitors are anti-hyperglycemic agents with well-characterized clinical efficacy in the treatment of T2DM. This class of drugs is also reported to exert pleiotropic effects, such as reductions in body mass, BP, and arterial stiffness, and the amelioration of aberrant endothelial function ${ }^{16-20,34-37}$. Furthermore, the outcomes of several recent clinical trials have indicated that SGLT2 inhibitors have cardio-renal protective effects in T2DM patients ${ }^{16-20}$. In the present study, we have demonstrated that the BP-lowering effect of dapagliflozin treatment is larger in patients with a high sodium intake than in those with a low sodium intake group, using BP measurements made at home.

The natriuretic effect of dapagliflozin is likely to contribute to this favorable BP-lowering effect. High dietary sodium intake promotes the expansion of extracellular fluid volume and increases cardiac output. To compensate for these hemodynamic changes and maintain BP, renal and peripheral vascular resistance falls because of an increase in the production of nitric oxide in patients with salt-resistant hypertension ${ }^{38}$. However, these compensatory mechanisms are impaired in patients with salt-sensitive hypertension ${ }^{38}$. Some previous studies have shown that the reduction in BP that is associated with dapagliflozin therapy has a natriuretic/diuretic-like effect, which is possibly associated with a reduction in plasma volume ${ }^{21}$. The BP-lowering effects of SGLT2 inhibitors are likely to be the result of the osmotic diuresis and mild natriuresis they induce ${ }^{21,22}$. The osmotic diuresis might contribute to an early reduction in BP by reducing extracellular fluid accumulation during SGLT2 inhibitor therapy ${ }^{39,40}$, whereas the natriuresis might play a larger role in the longer-term reduction in $\mathrm{BP}^{22}$.

In the present study, the reductions in UACR that were associated with dapagliflozin use were larger at weeks 8 and 16 in the HS group than in the LS group, but comparable at week 24. The UACR-lowering effect of SGLT2 inhibitors, which is partly associated with reductions in intraglomerular pressure, has been previously reported $^{41,42}$. Similarly, RAS inhibitors reduce albuminuria in patients with diabetic nephropathy because of a reduction in intraglomerular pressure, which is induced by the drug-induced dilation of efferent arterioles ${ }^{43}$. However, a post-hoc analysis of the Reduction of Endpoints in NIDDM with the Angiotensin II Antagonist Losartan (RENAAL) and Irbesartan Diabetic Nephropathy Trial (IDNT) trials demonstrated that the UACRand BP- lowering effects of ARB therapy are attenuated in patients with diabetic nephropathy and high sodium intake $^{15}$. Furthermore, the greater renal and cardiovascular protective effects associated with ARB therapy, compared with non-RAS inhibitor-based therapy, were also attenuated in patients with higher sodium intake ${ }^{15}$. In the present study, the natriuretic/diuretic effect of dapagliflozin may, at least partially, contribute to the finding that the reduction in UACR that was associated with dapagliflozin in the HS group was comparable to that in the LS group. Recently, a pooled analysis of data from two Phase 3 trials demonstrated that tofogliflozin also reduces UACR and clinic BP in Japanese patients with T2DM regardless of their basal daily sodium intake ${ }^{44}$. Our findings are consistent with these results ${ }^{44}$. It was recently reported that 6-week treatment with dapagliflozin did not reduce proteinuria compared with placebo in 53 proteinuric nondiabetic CKD patients in a randomized, double-blind, crossover trial (DIAMOND) ${ }^{45}$. However, dapagliflozin caused an anticipated acute dip in iohexolmeasured GFR, decreased body weight, and increased hematocrit, suggesting it caused reduced glomerular pressure and hemoconcentration with natriuresis (although BP was not significantly different between dapagliflozin and placebo treatment). It is possible the sample size was too small, and the follow-up period was too short, to detect significant changes in proteinuria in the DIAMOND study, as its design was based on studies in individuals with T2DM. All participants in the DIAMOND study had a nondiabetic kidney disease (IgA nephropathy, focal segmental glomerulosclerosis, hypertensive nephropathy, or other). Differences in underlying disease pathophysiology may have contributed to the inability to detect an antiproteinuric effect with dapagliflozin. Indeed, more recently, in the Dapagliflozin in Patients with Chronic Kidney Disease (DAPA-CKD) trial, which was a randomized, double-blind, placebo-controlled clinical trial with 4,304 CKD participants and median follow-up of 2.4 years, the risk of a renal composite outcome was significantly lower with dapagliflozin than with placebo among CKD patients regardless of the presence or absence of diabetes ${ }^{46}$.

In the present study, dapagliflozin had reduced eGFR to a similar extent in both groups at week 8, whereas the reduction was larger in the HS group than in the LS group at weeks 16 and 24. SGLT2 inhibitors block proximal tubular sodium reabsorption, which increases distal sodium delivery to the macula densa, thereby increasing tubulo-glomerular feedback, which leads to afferent arteriolar vasoconstriction, lower intraglomerular pressure, and less hyperfiltration in diabetic patients ${ }^{47}$. This is reflected in an initial transient dip in eGFR after SGLT2 inhibitor administration ${ }^{16,18-20}$. In the pooled analysis described above, high basal daily sodium intake positively correlated with the reduction in eGFR between the start and 52 weeks of tofogliflozin treatment ${ }^{44}$. The report concluded that the larger reduction in eGFR tofogliflozin-treated patients with higher sodium intake is due to the associated larger improvement in glomerular hyperfiltration ${ }^{44}$. In the present study, the larger reduction in eGFR in dapagliflozin-treated HS patients may be also attributed to the improvement in glomerular hyperfiltration. A difference in reduction of eGFR was not observed between the HS and LS groups at week 8 in the present study, whereas the pooled analysis showed that basal daily salt intake level was independently correlated with the change in eGFR from the $4^{\text {th }}$ week after tofogliflozin administration ${ }^{44}$. Differences in DKD stages among participants may have led to the discrepancy between our study and previous ones. In the previous pooled analysis, mean eGFR and median UACR were $83.9 \mathrm{ml} / \mathrm{min} / 1.73 \mathrm{~m}^{2}$ and $16.4 \mathrm{mg} / \mathrm{g} \mathrm{Cr}$ (IQR: 9.0, 46.0) at baseline ${ }^{44}$, respectively, suggesting their participants had earlier stages of DKD (where glomerular hyperfiltration may have been easier to improve) compared with participants in the present study.

Thus, the natriuresis-related hemodynamic effects of SGLT2 inhibitors play an important role in its BP-lowering and renoprotective effects. However, there are some differences between SGLT2 inhibitors and conventional 
diuretics. In general, diuretics such as loop diuretics and thiazides, which also have natriuretic effects, lower nocturnal $\mathrm{BP}$ more than daytime $\mathrm{BP}^{48}$. However, studies by ourselves and others have demonstrated that the reduction in BP induced by SGLT2 inhibitors persists throughout an entire day, but with a greater reduction in $\mathrm{BP}$ during the daytime than during the nighttime in T2DM patients ${ }^{23,49,50}$. In the present study, the reduction in nocturnal BP associated with dapagliflozin tended to be larger in the LS group than in the HS group, although the difference was not statistically significant. This may be partially attributed to the glucose-dependent nature of the glycemic control that is induced using SGLT2 inhibitors. The natriuretic effect of SGLT2 inhibitors may be greater during the daytime because of higher food and fluid intake. Conversely, diurnal hypertension is related to stress; therefore, an effect of SGLT2 inhibitors on sympathetic nerve activity may contribute to the greater reduction in diurnal $\mathrm{BP}^{51}$.

In addition to the difference in their BP-lowering effects, other diuretics, including thiazides and loop diuretics, do not have the robust cardio-renal protective effects that SGLT2 inhibitors were shown to have in the Empagliflozin Cardiovascular Outcome Event Trial in Type 2 Diabetes Mellitus Patients (EMPA-REG OUTCOME), the Canagliflozin Cardiovascular Assessment Study (CANVAS) Program, the Dapagliflozin Effect on Cardiovascular Events-Thrombolysis in Myocardial Infarction 58 (DECLARE-TIMI 58), and the Canagliflozin and Renal Events in Diabetes with Established Nephropathy Clinical Evaluation (CREDENCE) trials ${ }^{16-20}$. This may be because these conventional diuretics do not influence intraglomerular pressure directly ${ }^{3}$. Loop diuretics inhibit the $\mathrm{Na}^{+} / \mathrm{K}^{+} / 2 \mathrm{Cl}^{-}$cotransporter in the loop of Henle, which causes an increase in the delivery of sodium to the macula densa. However, in the macula densa, the reabsorption of sodium depends on the $\mathrm{Na}^{+}$/ $\mathrm{K}^{+} / 2 \mathrm{Cl}^{-}$cotransporter. Therefore, loop diuretics do not affect tubulo-glomerular feedback ${ }^{3}$, and other diuretics, such as thiazides, amiloride, and mineralocorticoid antagonists, act too distally to affect tubulo-glomerular feedback ${ }^{3}$. SGLT2 inhibitors inhibit not only SGLT2 but also $\mathrm{Na}^{+} / \mathrm{H}^{+}$-exchanger 3 (NHE3) activity ${ }^{52,53}$, by which approximately $30 \%$ of filtered sodium is reabsorbed in the proximal tubule, leading to natriuresis, which triggers tubulo-glomerular feedback ${ }^{3}$. It has also been reported that the effects of SGLT2 inhibitors on fluid distribution may differ from those of conventional diuretics ${ }^{54}$. These differences in pharmacological mechanisms explain the clinical favorable outcomes of SGLT2 inhibitors.

The limitations of the present study were as follows. The most critical limitation, sodium intake, as estimated using urinary sodium excretion on the basis of single-spot urine sampling, may have contributed to large variations in the data. Some studies reported that urinary sodium-to-creatinine ratios derived from spot urine sampling correlated weakly with those derived from 24-h urine collection ${ }^{55}$. Multiple reports suggest sodium intake calculated using the Tanaka formula is biased with overestimation at lower levels and underestimation at higher levels ${ }^{55-59}$. A method such as 24 -h urine collection may have provided a more accurate evaluation of sodium intake. Second, the study may have been underpowered to identify the differences in the effects of dapagliflozin according to sodium intake because the sample size calculation was based on the expected changes in ln-UACR ${ }^{23}$. Third, because the present study was a retrospective analysis of Y-AIDA study data, a cause and effect relationship could not be established. It is possible that sodium intake was only a confounding factor of other parameters affecting the reaction to SGLT2 inhibitors, because the HS group experienced significantly longer durations of diabetes and higher levels of HbA1c. Moreover, eGFR and BMI were higher in the HS group, although they were not significantly different at baseline. Other studies showed that SGLT2 inhibitors function more efficiently in patients with higher HbAlc levels and longer history of diabetes ${ }^{60}$. However, in the DECLARETIMI 58 trial, subgroup analysis of the renal composite outcome showed that favorable effects of dapagliflozin on renal composite outcomes in T2DM patients were consistent across baseline HbA1c levels and diabetes durations ${ }^{61}$. Regarding DKD patients in the CREDENCE study, reduction of risk for renal composite outcome with canagliflozin was also apparent in subgroups of patients with well-controlled diabetes, suggesting the effect of SGLT2 inhibitors on kidney function is unlikely to be mediated by further improvement of glycemic control ${ }^{62}$. Fourth, we cannot exclude the possibility that inclusion in the study resulted in participants changing lifestyle habits that influence nutrition, sodium intake, and glycemic control, all of which affect changes in albuminuria and BP. Fifth, extracellular fluid status was not assessed in the present study. Some previous studies have shown that the reduction in extracellular fluid volume achieved using SGLT2 inhibitors is transient ${ }^{40}$, and we did not assess whether dapagliflozin treatment reduced extracellular fluid volume throughout the present study period.

In conclusion, among Japanese DKD patients, there was no significant difference in reduction of UACR by dapagliflozin between the HS and LS groups. Furthermore, the BP-lowering effects of dapagliflozin, assessed using BP measurements made at home, were larger in the HS group than in the LS group, and this occurred alongside a larger reduction in eGFR, which suggests a dapagliflozin-induced improvement in glomerular relative hyperfiltration in the HS group.

\section{Data availability}

The datasets analyzed during the current study are available from the corresponding author on reasonable request.

Received: 28 August 2020; Accepted: 2 December 2020

Published online: 13 January 2021

\section{References}

1. Emerging Risk Factors Collaboration, Sarwar, N. et al. Diabetes mellitus, fasting blood glucose concentration, and risk of vascular disease: A collaborative meta-analysis of 102 prospective studies. Lancet. 375, 2215-22 (2010).

2. Thomas, MC., Cooper, ME. \& Zimmet, P. Changing epidemiology of type 2 diabetes mellitus and associated chronic kidney disease. Nat Rev Nephrol. 12, 73-81 (2016). 
3. León Jiménez, D., Cherney, D.Z.I., Bjornstad, P., Castilla-Guerra, L . \& González, J.P.M. Antihyperglycemic agents as novel natriuretic therapies in diabetic kidney disease. Am. J. Physiol. Renal Physiol. 315, F1406-F1415 (2018).

4. Vallon, V., \& Thomson, SC. Renal function in diabetic disease models: the tubular system in the pathophysiology of the diabetic kidney. Annu. Rev. Physiol. 74, 351-375 (2012).

5. Allen, TJ. et al. Glomerular filtration rate in streptozocin-induced diabetic rats. Role of exchangeable sodium, vasoactive hormones, and insulin therapy. Diabetes. 39, 1182-1190 (1990).

6. De Nicola, L. et al. Sodium/glucose cotransporter 2 inhibitors and prevention of diabetic nephropathy: targeting the renal tubule in diabetes. Am. J. Kidney Dis. 64, 16-24 (2014).

7. Tonneijck, L. et al. Glomerular hyperfiltration in diabetes: Mechanisms, clinical significance, and treatment. J. Am. Soc. Nephrol. 28, 1023-1039 (2017).

8. Mozaffarian, D. et al. Global sodium consumption and death from cardiovascular causes. N. Engl. J. Med. 371, 624-634 (2014).

9. Sacks, FM. et al. Effects on blood pressure of reduced dietary sodium and the Dietary Approaches to Stop Hypertension (DASH) diet. DASH-Sodium Collaborative Research Group. N. Engl. J. Med. 344, 3-10 (2001).

10. He, FJ. \& MacGregor, GA. A comprehensive review on salt and health and current experience of worldwide salt reduction programmes. J. Hum. Hypertens. 23, 363-84 (2009).

11. Whelton, PK. et al. Sodium reduction and weight loss in the treatment of hypertension in older persons: A randomized controlled trial of nonpharmacologic interventions in the elderly (TONE). TONE Collaborative Research Group. JAMA. 279, 839-846 (1998).

12. Williams, B. et al. 2018 ESC/ESH Guidelines for the management of arterial hypertension. Eur. Heart J. 39, 3021-3104 (2018).

13. Imanishi, M. et al. Sodium sensitivity related to albuminuria appearing before hypertension in type 2 diabetic patients. Diabetes Care 24, 111-116 (2001).

14. Horikawa, C. et al. Dietary sodium intake and incidence of diabetes complications in Japanese patients with type 2 diabetes: Analysis of the Japan Diabetes Complications Study (JDCS). J. Clin. Endocrinol. Metab. 99, 3635-3643 (2014).

15. Lambers Heerspink, H.J. et al. Moderation of dietary sodium potentiates the renal and cardiovascular protective effects of angiotensin receptor blockers. Kidney Int. 82, 330-337 (2012).

16. Wiviott, SD. et al.; DECLARE-TIMI 58 Investigators. Dapagliflozin and cardiovascular outcomes in type 2 diabetes. N. Engl. J. Med. 380, 347-357 (2019).

17. Zinman, B. et al.; EMPA-REG OUTCOME Investigators. Empagliflozin, cardiovascular outcomes, and mortality in type 2 diabetes. N. Engl. J. Med. 373, 2117-2128 (2015).

18. Wanner, C. et al.; EMPA-REG OUTCOME Investigators. Empagliflozin and progression of kidney disease in type 2 diabetes. $N$. Engl. J. Med. 375, 323-334 (2016).

19. Neal, B. et al.; CANVAS Program Collaborative Group. Canagliflozin and cardiovascular and renal events in type 2 diabetes. $N$. Engl. J. Med. 377, 644-657 (2017).

20. Perkovic, V. et al.; CREDENCE Trial Investigators. Canagliflozin and renal outcomes in type 2 diabetes and nephropathy. N. Engl. J. Med. 380, 2295-2306 (2019).

21. Lambers Heerspink, H.J., de Zeeuw, D., Wie, L., Leslie, B. \& List, J. Dapagliflozin a glucose-regulating drug with diuretic properties in subjects with type 2 diabetes. Diabetes Obes. Metab. 15, 853-862 (2013).

22. Kawasoe, S. et al. Mechanism of the blood pressure-lowering effect of sodium-glucose cotransporter 2 inhibitors in obese patients with type 2 diabetes. BMC Pharmacol. Toxicol. 18, 23 (2017).

23. Kinguchi, S. et al. Improved home BP profile with dapagliflozin is associated with amelioration of albuminuria in Japanese patients with diabetic nephropathy: the Yokohama add-on inhibitory efficacy of dapagliflozin on albuminuria in Japanese patients with type 2 diabetes study (Y-AIDA study). Cardiovasc. Diabetol. 18, 110 (2019).

24. Takahashi, H., Yoshika, M. \& Yokoi, T. Validation of two automatic devices: Omron HEM-7252G-HP and Omron HEM-7251G for self-measurement of blood pressure according to the European Society of Hypertension International Protocol revision 2010. Blood Press. Monit. 20, 286-290 (2015).

25. Kario, K. et al. Comparative effects of an angiotensin II receptor blocker (ARB)/diuretic vs. ARB/calcium-channel blocker combination on uncontrolled nocturnal hypertension evaluated by information and communication technology-based nocturnal home blood pressure monitoring-The NOCTURNE study. Circ J. 81, 948-957 (2017).

26. Tanaka, T. et al. A simple method to estimate populational 24-h urinary sodium and potassium excretion using a casual urine specimen. J. Hum. Hypertens. 16, 97-103 (2002).

27. Ogura, M. et al. Estimation of salt intake from spot urine samples in patients with chronic kidney disease. BMC Nephrol. 13, 36 (2012).

28. Katsuya, T., Ishikawa, K., Sugimoto, K., Rakugi, H. \& Ogihara, T. Salt sensitivity of Japanese from the viewpoint of gene polymorphism. Hypertens. Res. 26, 521-525 (2003).

29. Powles, J. et al. Global, regional and national sodium intakes in 1990 and 2010: A systematic analysis of 24 h urinary sodium excretion and dietary surveys worldwide. BMJ Open. 3, e003733 (2013).

30. Suckling, RJ., He, FJ., Markandu, ND. \& MacGregor, GA. Modest salt reduction lowers blood pressure and albumin excretion in impaired glucose tolerance and type 2 diabetes mellitus: A randomized double-blind trial. Hypertension. 67, 1189-1195 (2016).

31. Satoh, A. et al. Associations of socioeconomic status with prevalence, awareness, treatment, and control of hypertension in a general Japanese population: NIPPON DATA2010. J. Hypertens. 35, 401-408 (2017).

32. SPRINT Research Group, Wright, JT Jr. et al. A randomized trial of intensive versus standard blood-pressure control. N. Engl. J. Med. 373, 2103-2116 (2015).

33. Sciarretta, S., Palano, F., Tocci, G., Baldini, R. \& Volpe, M. Antihypertensive treatment and development of heart failure in hypertension: A Bayesian network meta-analysis of studies in patients with hypertension and high cardiovascular risk. Arch. Intern. Med. 171, 384-394 (2011).

34. Chilton, R. et al. Effects of empagliflozin on blood pressure and markers of arterial stiffness and vascular resistance in patients with type 2 diabetes. Diabetes Obes. Metab. 17, 1180-1193 (2015).

35. Pfeifer, M., Townsend, R. R., Davies, M. J., Vijapurkar, U. \& Ren, J. Effects of canagliflozin, a sodium glucose co-transporter 2 inhibitor, on blood pressure and markers of arterial stiffness in patients with type 2 diabetes mellitus: A post hoc analysis. Cardiovasc. Diabetol. 16, 29 (2017).

36. Shigiyama, F. et al. Effectiveness of dapagliflozin on vascular endothelial function and glycemic control in patients with early-stage type 2 diabetes mellitus: DEFENCE study. Cardiovasc. Diabetol. 16, 84 (2017).

37. Solini, A. et al. Dapagliflozin acutely improves endothelial dysfunction, reduces aortic stiffness and renal resistive index in type 2 diabetic patients: a pilot study. Cardiovasc. Diabetol. 16, 138 (2017).

38. Feng, W., Dell'Italia, L.J. \& Sanders, P.W. Novel paradigms of salt and hypertension. J. Am. Soc. Nephrol. 28, 1362-1369 (2017).

39. Iizuka, T. et al. Efficacy and safety of ipragliflozin in Japanese patients with type 2 diabetes: Interim outcome of the ASSIGN-K study. J. Clin. Med. Res. 8, 116-125 (2016).

40. Schork, A. et al. Effect of SGLT2 inhibitors on body composition, fluid status and renin-angiotensin-aldosterone system in type 2 diabetes: A prospective study using bioimpedance spectroscopy. Cardiovasc. Diabetol. 18, 46 (2019).

41. Kelly, M. S., Lewis, J., Huntsberry, A. M., Dea, L. \& Portillo, I. Efficacy and renal outcomes of SGLT2 inhibitors in patients with type 2 diabetes and chronic kidney disease. Postgrad. Med. 131, 31-42 (2019). 
42. Dekkers, C.C.J. et al. Effects of the SGLT-2 inhibitor dapagliflozin on glomerular and tubular injury markers. Diabetes Obes. Metab. 20, 1988-1993 (2018).

43. Ravera, M., Ratto, E., Vettoretti, S., Parodi, D. \& Deferrari, G. Prevention and treatment of diabetic nephropathy: The program for irbesartan mortality and morbidity evaluation. J. Am. Soc. Nephrol. 16(Suppl 1), S48-52 (2005).

44. Nunoi, K., Sato, Y., Kaku, K., Yoshida, A. \& Suganami, H. Renal effects of a sodium-glucose cotransporter 2 inhibitor, tofogliflozin, in relation to sodium intake and glycaemic status. Diabetes Obes. Metab. 21, 1715-1724 (2019).

45. Cherney, D.Z.I. et al. Effects of the SGLT2 inhibitor dapagliflozin on proteinuria in non-diabetic patients with chronic kidney disease (DIAMOND): A randomised, double-blind, crossover trial. Lancet Diabetes Endocrinol. 8, 582-593 (2020).

46. Heerspink, H.J.L. et al. Dapagliflozin in patients with chronic kidney disease. N. Engl. J. Med. 383, 1436-1446 (2020).

47. Cherney, DZ. et al. Renal hemodynamic effect of sodium-glucose cotransporter 2 inhibition in patients with type 1 diabetes mellitus. Circulation. 129, 587-597 (2014).

48. Uzu, T. \& Kimura, G. Diuretics shift circadian rhythm of blood pressure from nondipper to dipper in essential hypertension. Circulation 100, 1635-1638 (1999).

49. Baker, W.L. et al. Effects of sodium-glucose cotransporter 2 inhibitors on 24-hour ambulatory blood pressure: A systematic review and meta-analysis. J. Am. Heart Assoc. 6, e005686 (2017).

50. Kario, K. et al. 24-hour blood pressure-lowering effect of an SGLT-2 inhibitor in patients with diabetes and uncontrolled nocturnal hypertension: Results from the randomized placebo-controlled SACRA study. Circulation 139, 2089-2097 (2019).

51. Sano, M., Chen, S., Imazeki, H., Ochiai, H. \& Seino, Y. Changes in heart rate in patients with type 2 diabetes mellitus after treatment with luseogliflozin: Subanalysis of placebo-controlled, double-blind clinical trials. J Diabetes Investig. 9, 638-641 (2018).

52. Pessoa, T.D., Campos, L.C., Carraro-Lacroix, L., Girardi, A.C.C. \& Malnic, G. Functional role of glucose metabolism, osmotic stress, and sodium-glucose cotransporter isoform-mediated transport on $\mathrm{Na}+\mathrm{H}+$ exchanger isoform 3 activity in the renal proximal tubule. J. Am. Soc. Nephrol. 25, 2028-2039 (2014).

53. Vallon, V. \& Thomson, S.C. Targeting renal glucose reabsorption to treat hyperglycaemia: The pleiotropic effects of SGLT2 inhibition. Diabetologia 60, 215-225 (2017).

54. Ohara, K. et al. Effects of the sodium-glucose cotransporter 2 inhibitor dapagliflozin on fluid distribution: A comparison study with furosemide and tolvaptan. Nephrology. 24, 904-911 (2019).

55. Wan, E.R., Cross, J., Sofat, R. \& Walsh, S.B. 24-hour vs. spot urinary sodium and potassium measurements in adult hypertensive patients: A cohort validation study. Am. J. Hypertens. 32, 983-991 (2019).

56. Peng, Y. et al. A new approach is needed to evaluate 24-hour urinary sodium excretion using spot urines: A validation study in a Chinese child population. J. Am. Heart Assoc. 9, e014575 (2020).

57. He, F.J. et al. Formulas to estimate dietary sodium intake from spot urine alter sodium-mortality relationship. Hypertension 74, $572-580$ (2019).

58. Mill, J.G., Rodrigues, S.L., Baldo, M.P., Malta, D.C. \& Szwarcwald, C.L. Validation study of the Tanaka and Kawasaki equations to estimate the daily sodium excretion by a spot urine sample. Rev. Bras. Epidemiol. 18(Suppl 2), 224-237 (2015).

59. Rhee, M.Y. et al. Estimation of 24-hour urinary sodium excretion using spot urine samples. Nutrients 6, 2360-2375 (2014).

60. Uhrig, J.L. et al. Should baseline hemoglobin $A_{1 c}$ or dose of SGLT-2i guide treatment with SGLT-2i versus DPP-4i in people with type 2 diabetes? A meta-analysis and systematic review. J. Clin. Pharmacol. 60, 980-991 (2020).

61. Mosenzon, O. et al. Effects of dapagliflozin on development and progression of kidney disease in patients with type 2 diabetes: An analysis from the DECLARE-TIMI 58 randomised trial. Lancet Diabetes Endocrinol. 7, 606-617 (2019).

62. Cannon, C. et al. Evaluating the effects of canagliflozin on cardiovascular and renal events in patients with type 2 diabetes mellitus and chronic kidney disease according to baseline $\mathrm{HbAlc}$, including those with $\mathrm{HbAlc}<7 \%$ : Results from the CREDENCE trial. Circulation 141, 407-410 (2020).

\section{Acknowledgements}

This work was supported by grants from the Yokohama Foundation for Advancement of Medical Science; the Uehara Memorial Foundation; the Kanae Foundation for the Promotion of Medical Science; the Japan Society for the Promotion of Science, SENSHIN Medical Research; the MSD Life Science Foundation International; the Salt Science Research Foundation (18C4, 19C4, 20C4); The Cardiovascular Research Fund, Tokyo; the Strategic Research Project of Yokohama City University; the Japan Agency for Medical Research and Development (AMED); and by The Translational Research program, Strategic PRomotion for practical application of INnovative medical Technology (TR-SPRINT) from AMED.

\section{Author contributions}

S.K., H.W., K.A., T.Y., Y.T. and K.T. designed and conducted the research. S.K., H.W., Y.I., Y.K., K.A., U.O., T.Y., T.I., J.Y., G.Y., T.Y., K.H., Y.K., Y.T. and K.T. collected the data. T.M. and T.Y. analysed the data. S.K., H.W., K.A. and K.T. wrote the manuscript. All authors read and approved the final manuscript.

\section{Competing interests}

The authors declare no competing interests.

\section{Additional information}

Correspondence and requests for materials should be addressed to H.W., Y.T. or K.T.

Reprints and permissions information is available at www.nature.com/reprints.

Publisher's note Springer Nature remains neutral with regard to jurisdictional claims in published maps and institutional affiliations. 
(c) (i) Open Access This article is licensed under a Creative Commons Attribution 4.0 International cc) License, which permits use, sharing, adaptation, distribution and reproduction in any medium or format, as long as you give appropriate credit to the original author(s) and the source, provide a link to the Creative Commons licence, and indicate if changes were made. The images or other third party material in this article are included in the article's Creative Commons licence, unless indicated otherwise in a credit line to the material. If material is not included in the article's Creative Commons licence and your intended use is not permitted by statutory regulation or exceeds the permitted use, you will need to obtain permission directly from the copyright holder. To view a copy of this licence, visit http://creativecommons.org/licenses/by/4.0/.

(C) The Author(s) 2021 